\title{
Long-term outcome after STA-MCA anastomosis for moyamoya disease
}

\section{Susumu Miyamoto, M.D., Yoshinori Akiyama, M.D., Izumi Nagata, M.D., Jun Karasawa, M.D., Kazuhiko Nozaki, M.D., Nobuo Hashimoto, M.D., and Haruhiko Kikuchi, M.D.}

Department of Neurosurgery, Kyoto University Medical School, Kyoto; National Cardiovascular Center, Suita; Osaka Neurological Institute, Toyonaka, Japan

A long-term assessment was performed to determine the posttreatment clinical course of 113 patients with moyamoya disease. All patients sustained cerebral ischemic attacks and underwent superficial temporal artery-middle cerebral artery anastomosis with or without temporal muscle grafting. The follow-up duration was 3 to 24 years (mean $14.4 \pm 5.8$ [standard deviation]). Complete cessation of the ischemic episodes was obtained in 110 of 113 patients. One hundred patients were able to return to independent acitvities of daily living. Intellectual delays prevented 24 patients from engaging in an independent social life . Although intracranial bleeding is one of the common manifestations in moyamoya disease, hemorrhage was not detected in the 113 patients who underwent cerebral revascularization.

Key Words * moyamoya disease * superficial temporal artery-middle cerebral artery anastomosis * cesarean section

Moyamoya disease is a chronic progressive cerebrovascular occlusive disease that causes abnormal enlargement of perforating arteries (collateral pathways or moyamoya vessels) in the region of basal ganglia and thalamus. Most children with this disease suffer from cerebral ischemic symptoms that are usually induced by hyperventilation-associated actions such as crying, running, or even eating hot noodles. In contrast, approximately $50 \%$ of adult patients with moyamoya disease typically present with paraventricular hemorrhage with ventricular perforation, which has been attributed to hemodynamic stress placed on the abnormally enlarged moyamoya vessels. Cerebral revascularization procedures have been reported to improve the compromised cerebral blood flow, to reduce ischemic attacks, and to produce sufficiently good long-term results in most cases.[3-6,9] However, several issues remain controversial. If properly treated in childhood, can hemorrhage be avoided when the patient matures? Do headaches, which are a common symptom in moyamoya disease, have any relation to cerebral ischemic attacks? When female patients become pregnant, what is the best way to avoid cerebral bleeding during pregnancy and delivery? This long-term follow-up study was conducted to study these unresolved issues. 


\section{CLINICAL MATERIAL AND METHODS}

Since 1974, the authors have treated 248 patients with moyamoya disease who underwent superficial temporal artery to middle cerebral artery (STA-MCA) anastomosis and/or temporal muscle grafting (encephalomyosynangiosis [EMS]). To assess their posttreatment courses, a long-term follow-up study was undertaken. Because most of the patients live far from our institutes, mailed questionnaires were used for the assessment. Of 248 patients, 143 responded to the questionnaire. To protect patient privacy, the questionnaire could be answered anonymously. Twelve patients were excluded from this study because their follow-up period lasted no more than 3 years. Eighteen patients initially showed bleeding at onset. As a result, a long-term follow-up study was undertaken to assess posttreatment outcomes in 113 patients with ischemic onset.

The questionaire focused on issues of stroke recurrence, incidence of bleeding episodes, headaches, activities of daily living (ADLs), and the degree of intellectual delay in relation to the successful performance of ADLs. The ADL scale was divided into five classifications; excellent (no neurological deficit), good (independent with minor neurological deficit), fair (requiring some aid to perform ADLs), poor (mostly dependent), and dead. Intellectual delays were assessed in three categories: normal (possible to carry on a social life), minor (impossible to carry on an independent social life), and major retardation (impossible to carry out ADLs independently).

\section{RESULTS}

\section{Stroke Recurrence}

In 73 patients, transient ischemic attacks (TIAs) disappeared promptly after undergoing bypass surgery. In 37 patients, the incidence of TIAs gradually decreased postoperation and completely disappeared within 1 year. Thus, complete remission of the ischemic attacks was obtained in 110 patients. Residual TIAs persisted in three patients. Two patients experienced transient motor weakness in their lower extremities and one patient suffered from transient speech disturbance. These transient attacks occurred few times a year in each patient. There were no de novo intracranial hemorrhages. Ten patients described experiencing sporadic scintillating scotoma. One patient sustained periodic epileptic seizures.

\section{Activities of Daily Living and Intellectual Delay}

The ADL ratings were excellent in 76 patients, good in 24, fair in six, and poor in seven patients. The seven patients who experienced poor outcomes had already suffered such problems as pseudobulbar palsy, tetraparesis, and intellectual delay from major completed strokes before receiving surgical treatment. In the category of intellectual delay, 89 patients and/or their family members did not believe that they had any difficulties in carrying on an independent social life. Fifteen patients had difficulty carrying on an independent social life because of minor intellectual delays. The ADLs were limited in nine patients because of major intellectual delay.

\section{Headaches}

Forty patients complained of headaches even after their operations. Forty-two stated that they had suffered no incidence of headaches. Thirty-one respondents did not answer any of the questions regarding headaches. In 40 patients with headaches, the location of the pain was in the temporal or orbitozygomatic region in 21 patients, in the orbital or retroorbiral region in six, in both regions in four, and in other regions in nine patients. 
Headaches usually occurred early in the morning in 26 patients, in the afternoon in four, and at various times in 10 patients. Associated vomiting was noted by 19 of 40 respondents who complained of headache attacks. These headaches occurred several times a year in these 40 patients. No TIAs were found to be associated with headaches. Scintillating scotoma was associated with headaches in two patients.

\section{Pregnancy and Delivery}

Of 143 patients, 13 were pregnant and gave birth. Moyamoya disease was diagnosed in two patients after they suffered intracranial hemorrhage during late pregnancy. Five patients gave birth via transvaginal delivery without sustaining ischemic or hemorrhagic attacks prior to diagnosis and treatment. Five patients gave birth (transvaginal in two and cesarean section in three patients) after undergoing cerebral revascularization; no cerebrovascular attacks occurred during their pregnancies and deliveries.

\section{DISCUSSION}

Moyamoya disease is unique in that its presenting symptom is either ischemic or hemorrhagic. Although most pediatric patients suffer from ischemic attacks, adult patients may experience either type of onset, and some patients experience both. Cerebral revascularization surgery has been reported to be effective in improving ischemic symptoms and in stroke prevention.[3-6,9] The confusing variety of revascularization techniques can be divided into two categories: direct anastomotic bypass and indirect nonanastomotic bypass surgeries.[7,8,10] Indirect bypass procedures such as encephaloduroarteriosynangiosis or EMS are commonly performed because of their technical ease, although several authors have described cases in which patients are refractory to indirect bypass surgery.[1,5,7,8,10] To perform a direct bypass, microvascular anastomosis between small-caliber (usually under $1 \mathrm{~mm}$ in diameter) fragile vessels is required. As mentioned previously, several problems remain unresolved. It is not the purpose of this article to simply compare the two surgical procedures but to help to clarify the unresolved treatment and outcome problems through the long-term follow-up assessment in patients.

With regard to stroke recurrence and ADLs, the long-term outcome was satisfactory. Except for three patients who experienced sporadic TIAs, complete stroke disappearance was obtained in $110(97.3 \%)$ of 113 patients. The ability to live an independent life (excellent and good) was possible in $100(88.5 \%)$ of the 113 patients. However, the incidence of intellectual delay was unexpectedly high.[3] This study is not based on a quantitative analysis of intelligence but simply on anonymous mailed-in questionnaires. However, it is a fact that 24 patients $(21.2 \%)$ and/or their family members reported experiencing some difficulty in carrying on independent social lives, not because of motor skill deficits but because of intellectual deficits. In cases treated earlier in this long-term follow-up study, some patients had already become handicapped or intellectually delayed before admission.

Today, moyamoya disease is an entity well known to pediatric physicians, and magnetic resonance imaging and magnetic resonance angiography enable early diagnosis. Thus, most patients can be treated properly before suffering a completed stroke. However, the incidence of minor intellectual delay should be kept in mind. For treatment of anterior cerebral artery distribution, several surgical procedures such as STA to anterior cerebral artery anastomosis, omental graft, and galeal flap grafting have been described. These procedures have been performed chiefly to treat motor weakness in patients' lower extremities. Although not yet determined, surgical indications for the prevention of intellectual delay should be 
sought.

Forty (35.4\%) of 113 patients reported experiencing episodes of headaches. In 31 of 40 who complained of headaches, the pain was located in the orbital, periorbital, and temporal regions. Most patients (26 of 40) suffered headaches early in the morning, and approximately half (19 of 40 patients) experienced associated nausea and vomiting. It is important to note that the headaches occurred sporadically and were never associated with TIA. Thus we should reassue our patients that although headaches may occur even after cerebral revascularization, the episodes have no relation to cerebral ischemia.

Hemodynamic stress on moyamoya vessels enlarged to collateral vessels decreases after cortical perfusion is restored postsurgery. Theoretically, the incidence of hemorrhage in moyamoya vessels would decrease after a bypass operation. However, the role of bypass surgery to prevent rebleeding in patients who have already sustained a hemorrhage has been controversial.[2] There has been little statistical data on the transition from ischemic onset during childhood to hemorrhagic episodes once the patient has matured. We have personally treated six such adult patients. Although they were admitted because of intraventricular hemorrhage, they had a history of childhood ischemic episodes, which were conservatively managed without cerebral revascularization surgery. At the very least, this long-term follow-up study demonstrates that 78 patients with moyamoya disease who had been properly treated with STA-MCA anastomosis and EMS during childhood did not experience intracranial hemorrhage thereafter. In addition, 35 adult patients with ischemic onset also did not suffer intracranial hemorrhage after undergoing revascularization surgery.

Pregnancy and delivery are problematical for patients with moyamoya disease. Although five patients experienced uneventful pregnancy and transvaginal delivery, two untreated patients suffered intracranial hemorrhage during late pregnancy. Because the number of these patients is so limited, we cannot draw any definite conclusion as to whether transvaginal delivery is safe for patients with moyamoya disease. We personally believe, however, that uneventful transvaginal delivery is possible if the patient has already undergone surgery for cerebral revascularization and if the patient does not suffer from TIAs induced by hyperventilation. Cesarean section is recommended for untreated patients and for those with postoperative residual TIAs.

In conclusion, the long-term outcome in patients who have undergone STA-MCA anastomosis for the treatment of moyamoya disease was satisfactory with regard to ADL. The unsolved problems such as intellectual delay and the prevention of hemorrhage remain to be solved in the future.

\section{References}

1. Cahan LD: Failure of encephalo-duro-arterio-synangiosis procedure in moyamoya disease. Pediatr Neurosci 12:58-62, 1985/1986

2. Fujii K, Ikezaki K, Irikura K, et al: The efficacy of bypass surgery for the patients with hemorrhagic moyamoya disease. Clin Neurol Neurosurg 99 (Suppl 2):S194-S195, 1997

3. Ishikawa T, Houkin K, Kamiyama H, et al: Effects of surgical revascularization on outcome of patients with pediatric moyamoya disease. Stroke 28:1170-1173, 1997

4. Karasawa J, Touho H, Ohnishi H, et al: Long-term follow-up study after extracranial-intracranial bypass surgery for anterior circulation ischemia in childhood moyamoya disease. J Neurosurg:77:84-89, 
5. Matsushima T, Fujiwara S, Nagata S, et al: Surgical treatment for paediatric patients with moyamoya disease by indirect revascularization procedures (EDAS, EMS, EMAS). Acta Neurochir 98:135-140, 1989

6. Matsushima Y, Inaba Y: Moyamoya disease in children and its surgical treatment. Introduction of a new surgical procedure and its follow-up angiograms. Childs Brain 11:155-170, 1984

7. Miyamoto S, Kikuchi H, Karasawa J, et al: Pitfalls in the surgical treatment of moyamoya disease. Operative techniques for refractory cases. J Neurosurg 68:537-543, 1988

8. Mizoi K, Kayama T, Yoshimoto T, et al: Indirect revascularization for moyamoya disease: is there a beneficial effect for adult patients? Surg Neurol 45:541-549, 1996

9. Nakashima H, Meguro T, Kawada S, et al: Long-term results of surgically treated moyamoya disease. Clin Neurol Neurosurg 99 (Suppl 2):S156-S161, 1997

10. Touho H, Karasawa J, Ohnishi H, et al: Surgical reconstruction of failed indirect anastomosis in childhood Moyamoya disease. Neurosurgery 32:935-940, 1993

Manuscript received September 15, 1998.

Accepted in final form October 6, 1998.

Address reprint requests to: Susumu Miyamoto, M.D., 54 Kawahara-cho, Shohgoin, Sakyo-ku, Kyoto 606-8507, Japan. e-mail: miy@kuhp.kyoto-u.ac.jp. 